\title{
CORRECTIONS
}

\section{Publisher Correction: Establishing the role of diet in the microbiota-disease axis}

\section{Raylene A. Reimer(D)}

Nature Reviews Gastroenterology \& Hepatology (2019) https://doi.org/10.1038/s41575-018-0093-7

Published online 12 December 2018

In the version of this article published online and in print, the intestinal epithelial barrier depicted in Fig. 1 was drawn incorrectly with the cells oriented in the wrong direction. This error has been corrected for the HTML and PDF versions of the article.

https://doi.org/10.1038/s41575-019-0137-7 I Published online 25 March 2019 